\title{
Enhanced Vision to improve Safety in Robotic Surgery
}

\author{
V.Penza ${ }^{\mathrm{a}}$, S. Moccia ${ }^{\mathrm{a}, \mathrm{b}}$, E. De Momi ${ }^{\mathrm{c}}$, L. S. Mattos ${ }^{\mathrm{a}}$, \\ ${ }^{a}$ Advanced Robotics Department, Istituto Italiano di Tecnologia, Genoa, Italy \\ ${ }^{\mathrm{b}}$ Department of Information Engineering, Università Politecnica delle Marche, Ancona, \\ Italy \\ ${ }^{c}$ Department of Electronics, Information and Bioengineering, Politecnico di Milano, \\ Milan, Italy
}

\begin{abstract}
In the last decades, major complications in surgery have emerged as a significant public health issue, so the practical implementation of safety measures to prevent injuries and deaths in different phases of surgery is required. The introduction of novel technologies in the operating theater, such as surgical robotic systems, opens new questions on how much and in which way the safety can be further improved. Computer Assisted Surgery (CAS), in combination with robotic systems, can greatly help in enhancing the surgeons' capabilities providing direct patient and process-specific support to surgeons with different degrees of experience. In particular, the application of Augmented Reality (AR) tools could represent a relevant step towards safer clinical procedures, improving the quality of healthcare.

This chapter describes the main areas involved in an AR system, such as computer vision methods for identification of areas of interest, surgical scene description and safety warning methods. Recent advances in the field are also presented, providing as an example the Enhanced Vision System for Robotic Surgery (EnViSoRS): an AR system to provide assistance towards the protection of vessels from injury during the execution of surgical procedures with a commercial robotic surgical system.
\end{abstract}

Keywords: Safety in robotic surgery, Computer-assisted surgery, Stereo reconstruction, Semantic segmentation, Soft tissue tracking, Augmented reality 


\section{Introduction}

Minimal Invasive Surgery (MIS) has revolutionized the traditional open surgery technique reducing the invasiveness of the access to the surgical site by inserting surgical instruments and endoscope through few small incisions, reducing patient's trauma, risk of infections, and thus, improving the surgical outcome. However, despite the benefits, the uptake of MIS surgery has been slow due to some limitations, including limited surgeons' maneuverability, reduced haptic and depth perception, limited freedom of movement due to the single endoscope port access and limited field of view of the surgical scene. Even if the advent of Robotics in Minimally Invasive Surgery (RMIS) has allowed to overcome many of these drawbacks, the core of the surgery still relies on the surgeons' degree of expertise and experience, and on their ability, for example, to mentally fuse preoperative information intraoperatively, making the outcome of the surgery vary according to the surgeons' skills.

Computer Assisted Surgery (CAS) can greatly help in enhancing the surgeon's capabilities providing direct, patient and process-specific support to surgeons with different degrees of experience, and its combination with surgical robotic systems would be valuable in surgical safety improvements. Computer assisted technologies have been developed to support different stages of the treatment procedure. During the pre-operative phase, Virtual Reality (VR) can help the surgeon to diagnose the disease and plan the treatment. Different techniques have been established to build 3D virtual models of the patient's anatomy from, for example, Digital Imaging and Communications in Medicine (DICOM) data. The virtual exploration of such models can assist the surgeon during the preliminary phase of a surgical procedure through interactive and visual planning of the operative strategy [1].

During the intra-operative phase, Augmented Reality (AR) systems can enhance surgical awareness and provide a more comfortable and efficient environment for the surgeon. This involves fusing the pre-operative information and the surgical plan with the intra-operative visualization of the surgical field $[2,3]$. The process of precisely adapting the virtual 3D model to match the patient's real anatomy is called registration. This is the key to create an AR environment and intraoperative navigation tools. By highlighting target structures and anatomical relationships through a modular virtual transparency, AR allows looking inside closed cavities or through solid structures [4,5]. Moreover, since in MIS or RMIS surgeons cannot rely on the "sense of touch" to identify major structures (such as tumor, vessels, etc.) or orient the surgical resection, AR can help by providing intra-operative visualization of such structures, contributing to reduce also the time required in the operating room [6].

Despite continuous progress over recent years, the registration of multimodal pre-operative patient data in soft-tissue surgery is still highly challenging as the anatomy undergoes significant changes between the data acquisition (pre-operative) phase and the surgical procedure (intra-operative). This is due to different factors [7]: (i) different pose of the patient with respect to the pose in which the pre-operative image was taken; (ii) $\mathrm{CO}_{2}$ abdominal insufflation for increasing the working volume (pneumoperitoneum); (iii) instrument tissue interaction; (iv) heart beat; (v) breathing. Considering all these aspects, the application of AR 
[Insert Figure 1 here]

Figure 1 Overview of the main areas involved in an Augmented Reality (AR) system to improve safety in surgery.

in minimally invasive surgery still presents a number of open issues which are the focus of current research.

This chapter is intended to give an overview of the main areas involved in an AR system for surgical applications, as shown in Fig. 1. In Section 2, general aspects of safety in surgery are tackled, focusing the attention on what a CAS system can provide in terms of safety to RMIS. Section 3 describes the different modalities for the selection and identification of a region of interest. The core techniques involved in the surgical scene understanding are detailed in Section 4. Section 5 describes different existing AR modalities to display the safety information to the surgeon. A practical example of an implementation of an AR system aimed at improving the safety in abdominal RMIS is presented in Section 6, and conclusions are presented in Section 7.

\section{Safety in Robotic Minimally Invasive Surgery}

According to the World Health Organization (WHO), the number of major surgical operations performed in 56 countries in 2004 was estimated between 187-281 million. It can be seen as approximately one operation per year for every 25 human beings alive [8]. Such a volume implicates complications in the surgical process that are documented as a major cause of injuries and death worldwide. In industrialized countries, the rate of major complications has been documented to occur in 3-22\% of surgical procedures, with a death rate between $0.4-0.8 \%$ [9]. Although the improvements in surgical safety knowledge, nearly half the adverse events in these studies were determined to be preventable. Consequently, surgical safety has emerged as a significant global public health concern.

With the campaign "Safe Surgery saves Life" between 2007 and 2009, the WHO has promoted the use of surgical safety checklist, a 19-item checklist that should be completed at the crucial steps of a surgical procedure: before induction of the anesthesia, before skin incision and before patient leaves the operating room. Its pilot study, conducted in eight hospitals around the world, reported a decrease of mortality and complications of $48 \%$ and $37 \%$, respectively [10]. Even if the reason of the improvement in safety is not clearly evincible, different studies demonstrated improvements in team communication, teamwork, compliance with prophylactic antibiotic administration and monitoring prior to induction of anesthesia [11].

Despite these efforts, major complications during surgery remain a problem. Focusing the attention only to laparoscopic procedure, [12] proposes a classification of major 
complications according to six different phases of the procedure: (i) Patient identification, (ii) Anesthesia and positioning, (iii) Abdominal entry and port placement, (iv) Surgery, (v) Postoperative recovery, (vi) Counselling. It is reported that more than $50 \%$ of complications occur during the abdominal entry and port placement (phase iii), where vascular, intestinal, urinary tract injury and gas embolism are the very common. Vascular injuries can occur also during surgery (phase iv), being the most serious intra-operative laparoscopic complication with a $9-17 \%$ mortality rate $[13,14]$. Risk factors are attributed to the surgeon's skills, instrument sharpness, angle of insertion, patient position, degree of abdominal wall elevation and volume of pneumoperitoneum [12].

In the last decades, new technologies in the robotics field have been introduced with the purpose of improving the outcome of the surgical procedures. Specifically, RMIS has demonstrated to improve precision, ergonomics and reduce fatigue and stress with respect to laparoscopic procedures. An example of a surgical robotic platform currently on the market is the da Vinci ${ }^{\mathrm{TM}}$ surgical system (Intuitive Surgical, Inc., Sunnyvale, CA), which is having a strong impact on the healthcare: by 2016 there were more than 3,700 systems worldwide and more than 3,000,000 operations performed [15,16]. Even if the surgeon can rely on such advanced technologies, the core of the surgical procedure is still under his/her total control. In fact, robotic assistance can help the surgeons in improving their performance while performing important and delicate actions, such as, for example, suturing. However, the intraoperative decision process is strongly dependent on their ability to dynamically fuse the preoperative patient-specific information into the information coming from the surgical scenario [17]. These tasks are made more difficult by the fact that the only feedback the surgeon receives from the surgical site is the video coming from the endoscope, whose field of view is limited by the characteristics of the camera and by the constrains imposed by the access point, which does not allow visualization from different perspectives.

The most promising solution for safer surgical procedures with a minimal rate of major complications consists in exploiting assistive technologies. The role of such technologies lies in enhancing the surgeon's capabilities during surgery, by providing information from preoperative phase, monitoring the surgical site and warning the surgeon in case of situations that can lead to adverse events.

\section{Identification and Definition of the Structure of Interest}

Depending on the surgical intervention, an AR application can be used to augment different targets, such as tumors, vessels, nerves or other anatomical structures. These structures can be semi-automatically extracted from anatomical pre-operative images during the pre-operative phase or can be manually identified intra-operatively on the endoscopic image. Hereafter, different methods for the two approaches will be described. 
[Insert Figure 2 here]

Figure 2 On the left $(a, b)$, an example of Volume Rendering from a CT scan of the abdomen. On the right, an example of Surface Rendering (c), with details of the 3D models of the liver and kidney $(d)$.

\subsection{Semi-Automatic Pre-0perative Identification}

The Structure of Interest (SOI) can be identified and extracted during the pre-operative phase in the form of a 3D virtual model built from pre-operative imaging. The most used imaging systems are Magnetic Resonance Imaging (MRI) and Computerized Tomography (CT), due to the high resolution they can provide. The two methods used to generate 3D organs models rendering are:

- Volume Rendering. Volume rendering allows 3D visualization of all structures represented in the medical images simultaneously, as shown in Fig. 2. Ray casting is one of the most popular techniques as it allows a very precise representation of the data. Virtual rays projected from the camera are used to traverse the 3D data, performing a weighted summation of the information of color and opacity associated with every voxel hit along the ray [18]. Therefore, the advantage of not requiring manual interaction of the user to delineate the structure to render in the $3 \mathrm{D}$ model turns into a limitation for $\mathrm{AR}$ applications, since the 3D model of different organs cannot be independently visualized and manipulated.

- Surface Rendering. Surface rendering allows the visualization of 3D organ models separately (see Fig. 2). As opposed to volume rendering, the first step for surface rendering involves manual user interaction for segmentation of the SOI. The segmented area can be obtained using different classic techniques such as thresholding, region growing, active contours, clustering and classifiers, while more advanced methods include Markov Random Fields (MRF), Artificial Neural Networks (ANN) and Statistical Shape Models (SSM) $[18,19,20])$. The segmentation is then exploited to convert the CT scan volumetric information into a polygonal mesh. The most common technique is the marching cubes [21].

\subsubsection{Registration}

With registration, the pre-operative organ anatomy is aligned with the intra-operative view [18]. The registration process is described by a mathematical operator called transformation, which can be either rigid or deformable:

1. Rigid registration: The transformation is described by six degrees of freedom, three for rotation and three for translation, assuming the rigidity of the scene. The most used rigid registration algorithm is Iterative Closest Point (ICP) [22,23]. 
2. Deformable registration: The transformation is described using six up to an infinite number of degrees of freedom, since the deformation model can be defined as complex as desired. A completed overview of deformable registration methods used in the medical field is reported in [24]. One of the most popular deformable transformations is based on vector fields $[25,26,27]$.

Both rigid and deformable registrations can be performed by a:

- Manual approach: The registration process is performed by an expert and relies on manual input. This strategy can be used with other strategies, for example to initialize surface- or volume-based registration algorithms. The manual approach is strongly affected by the quality of its user interface and by the operator's degree of expertise.

- Point-based approach: The registration is performed between two sets of corresponding points, one from the pre-operative view, one from the intra-operative scene. Point-based approaches require the identification of anatomical landmarks or artificial markers (usually attached to the organs and acquired with the help of a tracking system) visible both pre- and intra-operatively. In point-based approaches, the limitations are related to the need to visualize the same markers and to the tracking system inaccuracy. If the markers are manually identified, the degree of expertise of the user affects the quality of the procedure.

- Surface-based approach: In surface-based approaches, the reconstructed surface of the intraoperative scene (see Section 4.2) is registered to the surface of the pre-operative 3D model. The registration strongly depends on the accuracy of the reconstructed surface and it is reliable only for visible surfaces, as no assumption is made on the underlying structure. The integration of biomechanical models in the registration process can alleviate this issue, but the parameters of such models have to be determined.

- Volume-based approach: The volume-based approach is often adopted when there is an intra-operative imaging system available to retrieve the intra-operative 3D anatomy (such as cone-beam CT or 3D Ultrasound (US)). Unfortunately, such equipment is not always available in the Operating Room (OR). However, when it is, pre-operative models can be deformed according to biomechanical models to mimic the intra-operative surface.

\section{[Insert Figure 3 here]}

Figure 3 Example of three different modalities for the intra-operative selection of an area of interest. From left to right, (a) touch screen monitor, (b) graphical tablet, and (c) the control handle of a surgical robot. 


\subsection{Manual Intra-Operative Selection}

In the case it is not possible to identify the SOI pre-operatively, or this is not convenient, it is still possible to intra-operatively select a $2 \mathrm{D}$ area surrounding the SOI using the endoscopic images. The selection can be performed, for example, by means of a touch screen monitor, a graphical tablet or the robotic handle in case of surgery assisted by a robotic system $[28,29]$, as shown in Fig. 3. In this case, there is no need for registration with the intraoperative view, since the selection of the SOI is intra-operatively done.

\section{Surgical Scene description}

This section will introduce modern computer vision methods for surgical scene description. They perform such task by classifying the tissues in the field of view, retrieving their 3D surface information and tracking their deformations.

\subsection{Semantic Segmentation}

Intra-operative tissue classification plays a fundamental role in different clinical fields, such as laryngology [30,31], dermatology [32], oncology [33], ophthalmology [34], and neurosurgery [35]. Tissue classification is performed for diagnosis, treatment planning and execution, and for treatment outcome evaluation and follow up. The importance of intraoperative tissue classification is supported by the gradual but constant introduction in clinical practice of medical technologies aimed at enhancing the visualization of tissues, such as multispectral [36], narrow-band [37], and spectroscopy imaging [38].

Automatic image-based classification of tissues is a valuable solution to provide surgeons with decision support or context awareness intra-operatively. Despite the fact that automatic or semi-automatic tissue classification approaches have been proposed by the Surgical Data Science (SDS) community, several technical challenges are still present, hampering the clinical translation of the proposed methodology to clinical practice. Indeed, performing robust and reliable classification is not trivial due to the high inter- and intra-patient variability, and to noise in the images, varying illumination level, and changing camera pose with respect to the tissues. Nonetheless, Machine Learning (ML) strategies have recently shown to provide robust, reliable and accurate tissue classification for decision support and context awareness during interventional-medicine procedures.

ML models for tissue classification typically (i) apply automated image analysis to extract a vector of quantitative features to characterize the relevant image content and (ii) apply a pattern classifier to determine the category to which the extracted feature vector belongs (e.g., malignant / healthy tissue) $[39,40]$.

Intensity-based features aim at encoding information related to the prevalent intensity components in the image. Intensity features are mainly based on intensity histogram, mean, variance and entropy [41] and are commonly combined with textural features, which encode tissue appearance, structure and arrangement [42]. 
[Insert Figure 4 here]

Figure 4 Example of semantic segmentation of an endoscopic image of a phantom of abdomen. On the left, the RGB image, on the right the same image augmented with the tissue classification information obtained from a semantic segmentation algorithm [76].

Textural features include local binary pattern [43], gray-level co-occurrence matrix [44], and histogram of oriented gradients [45]. This class of features has been successfully used for several applications, such as tissue classification in gastric [46] and colorectal images $[47,48]$.

Other popular features are obtained with filtering-based approaches. Filtering approaches build template-filters that correlates with tissue structure in the image. Common approaches are matched-filtering [49] and wavelets [50], which have been widely used for vessel detection and localization [51] and polyp classification [52]. Similarly, derivative-based approaches build derivative-filters to extract image spatial derivatives, such as gradient and Laplacian, e.g. to highlight tissue edges $[53,54]$.

Recently, learned features have been proposed and successfully used for tissue classification [55,56]. Learned features refer to features that are automatically extracted from the image [57]. The most popular approach to automatic feature learning is using convolutional neural network, which showed remarkable performance in classifying skin cancer [55] and predict cardiovascular risk factors from retinal fundus photographs [56].

As for pattern classifiers, several solutions have been introduced in the last decades and an extensive review can be found in [58]. First attempts were based on statistical approaches (i.e. naive Bayes [59], Bayesian networks [60] and Bayes classifiers [61]) and instance-based learning [62]. Applications for tissue classification include [63,64]. Similarly, perceptronbased algorithms [65], have been widely used, e.g. for polyp detection in endoscopic images [66,67,68]. Logic-based algorithms [69,70] and support vector machines [71] are probably among the most widely used classifiers. These algorithms showed promising performance for tissue classification in several fields (e.g, $[30,72,73]$ ). More recently, deep learning for tissue classification drew the attention of the SDS community. Examples include skin-cancer classification [55], polyp detection [74], retinal image analysis [56], and vessel segmentation [75], where large and labeled datasets are publicly available for deep-learning model training.

An example of semantic segmentation for AR application can be found in [76], where the awareness of the scene is promoted by a confident multi-organ semantic segmentation, exploited to recognize different tissue textures on the endoscopic images (see Fig. 4). 


\title{
[Insert Figure 5 here]
}

\begin{abstract}
Figure 5 Scheme representing the main steps involved in dense stereo imaging: Stereo Images as input, Undistortion to remove possible distortions due to lenses imperfection, Rectification to align the pixels on the same epipolar lines, Stereo Correspondence to compute the match between the stereo images and Triangulation to compute the depth information. The output is the $3 D$ reconstruction of the surgical surface.
\end{abstract}

\subsection{Surgical Scene Reconstruction}

Recovering the surgical scene surface during laparoscopic surgical interventions is an essential step for surgical guidance and AR applications. It can be used for different purposes: to register pre-operative model on intra-operative images, to build a 3D map of the surgical site or to compute the geometrical relationship between the tissue and the surgical instruments $[77,78]$.

Different image modalities can be used to acquire detailed information about the tissue morphology, such as ultrasound (US), Computer Tomography (CT) and Interventional Magnetic Resonance Imaging (iMRI). However, issues related to real-time performance, healthy risks and high costs disfavor the use of such technologies in the intra-operative surgical scenario. This situation favors the use of intra-operative endoscopic images to perform on-line estimation of tissue surfaces through methods of depth estimation [79]. These methods can be divided into two categories [80]: passive methods that rely on the image information only, and active methods that make use of a light projection into the environment. Passive methods include stereoscopy [81,82,83], monocular Shape-from-X (SfX) and Simultaneous Localization and Mapping (SLAM) [84,85]. Active methods are mostly based on structured light and Time-of-Flight (ToF). A review of state-of-the-art methods for 3D reconstruction in laparoscopic surgery is reported in [86], and a comparative validation between the most relevant methods is presented in [87].

Since the use of passive methods does not require any change to the existing surgical room setup, stereoscopy is one of the most common methods for the reconstruction of the surgical scene. This approach is inspired by the stereo imaging capability of eyes. By finding correspondences between points that are seen by one eye and the same points as seen by the other eye, our brain is able to compute the 3D location of the points. Similarly, computers can process a pair of stereo images captured by a laparoscope and compute the 3D surface of the surgical field. The left-right correspondences can be computed in a sparse manner, where several salient regions (features) are detected and matched. Alternatively, a dense reconstruction can be obtained by matching every pixel of the stereo images. We report, as an example, the main steps involved in dense stereo imaging (see Fig. 5):

- Undistortion. The image acquired by the cameras are often affected by distortions, typically divided in radial and tangential, caused by the lens properties. The undistortion process is able to represent the image as a planar projection. 
- Rectification. In order to simplify the stereo-correspondence methods, the images are rectified thanks to a left-right calibration process. This computation ensures that equivalent pixels between left and right images lay in the same row of the image.

- Stereo-Correspondence. Stereo correspondence is the process of identification of the difference in position of an object represented by the stereo images, by finding the matching between each pixel of the left and the right images (iml and imr). This information is called disparity (d). The process can be divided in four steps, as stated in [88] and shown in Fig. 6:

1. Matching cost computation: exploits methods to calculate the similarity between two pixels, or windows around the pixels. For example, correlation (Normalized Cross Correlation (NCC)), intensity difference (Absolute Difference (AD), Squared Difference (SD)) or rank (Rank Transform, Census Transform) [89].

2. Aggregation cost computation: The aggregation of the matching cost is done by using the information from a window surrounding the target pixel. As an example, the Sum of Absolute Differences (SAD) is calculated for a pixel $(i, j)$ as described by Eq. 1 .

$$
S A D(i, j, d)=\sum_{\substack{h=-m / 2 \\ k=-n / 2}}^{m / 2} I_{l}(i+h, j+k)-I_{r}(i+h-d, j+k)
$$

where $I l$ and $I r$ is the intensity of each pixel of $i m l$ and $i m_{r}$ respectively, and $\mathrm{d}$ is the disparity.

3. Disparity computation: The computation of the disparity value at each pixel consists in choosing the disparity associated with the minimum of the aggregation cost value. An example of local computation is the Winner Takes All (WTA) strategy [88], which selects the pixel with the minimum aggregation cost value as best match. Other techniques use global or semi-global information in order to optimize the result of the disparity computation [88].

\section{[Insert Figure 6 here]}

Figure 6 Pipeline of the main steps involved in stereo-correspondence process.

4. Disparity refinement: Different strategies can be used to reduce the number of incorrect values (due to an incorrect matching). As an example, a Left-Right Consistency (LRC) check, can be performed in order to invalidate half-occluded pixels, i.e., objects viewed in one image but not in the other. Sub-pixel refinement is applied 
to avoid separated layers in the reconstructed surface resulting from pixel-level precision [90]. A speckle removal filter can also be applied in order to remove small artifacts, i.e., regions of large and small disparities that can be generated near the boundaries of shapes.

- Triangulation. The $\mathrm{Z}$ coordinate of the points, also called depth, can be computed as:

$$
\operatorname{depth}(i, j)=\frac{(f \cdot s)}{\operatorname{disparity}(i, j)}
$$

where $i$ and $j$ are respectively rows and columns of the image, $s$ is the baseline between the stereo cameras, $f$ is the camera focal length. See graphical representation in Fig. 5.

The 3D surface reconstruction of surgical endoscopic images is still an active area of research due to some challenging aspects that include: dynamic and deformable environment, many texture-less or homogeneous texture areas, specular highlights, smoke and blood produced during the intervention, and occlusions introduced by the surgical tools [91]. Moreover, the application of 3D surface reconstruction in surgery has to fulfil high requirements in terms of accuracy and robustness in order to ensure patient safety.

\subsection{Tissue Tracking}

In order to measure intra-operative tissue movements, computer vision and image processing algorithms have been exploited to track soft tissue areas relying only on the image characteristics [91]. Tissue tracking can be approached either from tracking or detection perspectives.

Tracking-based algorithms estimate tissue motion using feature matches between successive frames. The tracking features are identified on the tissue surface during an initialization phase. Since this method relies on the uniqueness of the features, the definition of the features strongly affects its reliability. For this reason, different image features have been explored, such as gradient, color and textural features [92], where each technique relies on different characteristics of the image. The main advantages of this approach are that it just requires an initialization, it is computationally cheap, and it produces smooth trajectories. On the other hand, it is prone to drift due to the error accumulation during runtime because of image noise and computational approximations. It also typically fails if the object is partially occluded or disappears from the camera view.

Detection-based approaches estimate the location of the tissue at every frame independently, typically relying on local image features or sliding windows. The steps of feature-based approaches [93] usually are: (i) feature detection, (ii) feature matching, and (iii) model fitting. Sliding window-based techniques [94] use a window to scan the input image, and typically use a classifier to decide whether the underlying patch contains the object to 
track or background. As opposed to the tracking-based techniques, the detectors do not present drift problems and are robust against total or partial occlusions, or when the tracked area disappears from the camera point of view. However, the use of a classifier requires an offline training phase and therefore cannot be applied to unknown objects.

The implementation of algorithms for soft tissue tracking that work robustly for a long period is still a challenging task. This is due to several problems related to the characteristics of endoscopic images. In order to reach the goal of robust long-term soft tissue tracking, the following issues have to be dealt with: (i) tissues are constantly subject to deformations due to breathing, heartbeat and instrument-tissue interactions; (ii) camera movements cause blurring and changes in scale and orientation of the object to be tracked; (iii) camera movement and instrument-tissue interaction can cause partial or total occlusion of the tracked tissue, even for a long period of time; (iv) the tissue may change its appearance, thus making the appearance from the initial frame irrelevant; (v) tissue images often exhibit considerable specular reflections caused by the wet tissue surfaces, and endocavity lighting creates dynamic lighting conditions.

\section{Safety Warning methods}

The methodological and technological advancements presented in the previous sections allow moving towards a complete understanding of the surgical scene, coupling the contributions of computer vision and robotic systems to empower the surgeon with novel capabilities that increase context awareness. Providing additional information to the surgeon can potentially help in preventing complications and thus improve surgical safety. There are multiple ways to present safety information to the surgeon, including visual feedback (i.e. AR features) and/or haptic feedback (i.e. active constraints), as described in the following sections.

\subsection{Augmented Reality Visualization}

AR techniques allow the rendering of safety information directly on the surgeon's view. The modality selected for AR depends on the surgical setup, i.e., on the way the surgeon observes the surgical scene: direct visualization, microscope visualization, or endoscopic visualization. Basic AR modalities, as described in [18], include:

- Projection on patient. This modality consists in projecting an image directly on the patient's body. This can give very good results when working with $2 \mathrm{D}$ features such as insertion points or other surface landmarks. However, the correct representation of 3D information is highly dependent on the surgeon's tracking, which is required to correct the perspective of the AR projection.

- Optical see-through. It is possible to render the AR information onto semi-transparent surfaces allowing the simultaneous visualization of virtual information and the real scene. The projection surface can be statically mounted in front of the scene, or on a 
wearable device. In the first case, the projection surface could interfere with the surgical instruments, while the second can cause discomfort. As an advantage with respect to the previous technique, the optical see-through solves the problem of occluding the projection.

- Video see-through. Alternatively, the rendering of the AR information can be done on a video streaming captured from the real surgical scenario using a camera. This technique has been increasingly explored in the last years due to the technological advances in Head-Mounted Displays (HMD) and handheld devices.

- Static video display. Finally, the representation of the surgical scene with AR information can be done on a statically mounted display receiving a video streaming from the surgical scenario. The video display can be a classical monitor, or the 3D display of a remote console such as the da Vinci ${ }^{\mathrm{TM}}$ surgical system.

Apart from visualization method, the kind of information transmitted to the surgeon is also important. This depends on the kind of surgery, the available information and the kind of assistance the system is designed to offer. For example, the AR information may represent:

- Pre-operative information. The most direct use of $\mathrm{AR}$ is to render the pre-operative information directly on the real images. This can help the surgeon to identify anatomical structures, such us veins or tumors. However, the accuracy of the overlay between the $\mathrm{AR}$ and the real scene can affect significantly the advantages of this technique.

- Processed information. It is also possible to transmit to the surgeon information obtained from some computational analysis of the surgical field. For example, in [95] the authors implement a supervisor for laser phono microsurgery where the AR system displays a 2D map representing the estimated tissue temperature and laser cutting depth. This information is displayed on a corner of the video streaming, not affecting the visualization of the surgical scenario, but providing significant information to the surgeon about the laser procedure.

[Insert Figure 7 here]

Figure 7 Overview of EnViSoRS system: Enhanced Vision System to improve Safety in Robotic Surgery. The system is integrated into dVRK system (WPI and Johns Hopkins University). From the console the surgeon can (i) select the safety area using a stylus and a graphics tablet, (ii) see the safety volume overlaid on the images, and (iii) see a graphical gauge that provides information regarding the tissue-instruments distance. 


\subsection{Active Constraints}

The idea of adding a sensory overlay (i.e. active constraints/ virtual fixtures) during the interaction between the human user and the robot was firstly introduced by [96]. Different constraint modalities were classified in [97] as regional/guidance, attractive/repulsive, unilateral/ bilateral, dynamic/static in a comprehensive review paper, listing research work published before 2014. Recent publications have been focused on the constraint design modalities, enforcement [98] (focusing on improving the interaction performances and the stability of the control approach) and application to laser surgery [99]. Some authors introduced constraints in a co-manipulation paradigm for keyhole surgery [100] or for bone drilling [101].

\section{Application in Abdominal Surgery: EnViSoRS system}

After the panoramic on different computer vision methods and safety warning visualization techniques so far shown, this section is intended to provide an example of application of such methods presenting the Enhanced Vision System for Robotic Surgery (EnViSoRS), a system that aims to minimize the risk of intra-operative bleeding during abdominal RMIS [29]. The system includes AR features that provide warning in case the robotic instruments get close to a user-defined Safety Area (SA), which can be defined intraoperatively by manually marking the tissue to be protected using graphic overlays. The system tracks the SA in real-time using a novel algorithm robust against common events happening during a surgical procedure, such as (i) camera movements, (ii) tissue deformations, and (iii) field of view occlusions due to smoke or to the presence of surgical instruments.

The core of EnViSoRS is based on novel computer vision algorithms aimed at retrieving the surgical scene information and processing such information to monitor the distance between the surgical instruments and the surface to protect in the 3D space. An overview of the system is shown in Fig. 7.

The system consists in the following five steps:

- Image Pre-Processing. The first necessary step is the calibration of the endoscopic stereo cameras. The extracted camera parameters are exploited to correct image distortions and perform rectification, which facilitates the search for stereo correspondences needed for the 3D reconstruction method. Specular highlights (identified as bright regions with low saturation and high intensity) are eliminated to prevent errors in feature tracking and 3D reconstruction computation. 
[Insert Figure 8 here]

Figure 8 Example of AR visualization of EnViSoRS. Top left, safety area selection on the endoscopic video image. Top right and bottom images represent three different instants of the safety system displaying the safety volume as a green ellipsoid, and the gauge indicating the distance between the surgical tool and the 3D surface of the protected area (further distances indicated by green segments, closer distances indicated by red segments).

- Safety Area Definition: EnViSoRS, at this stage of the development, does not need any information from pre-operative planning. At any moment during the surgery, the surgeon can define the SA as a 2D polygon on the endoscopic image using a graphics tablet and a stylus, as shown in Fig. 7 and Fig. 8. In our configuration, the tablet (WACOM Bamboo Pen and Touch Tablet) was placed directly on the da Vinci ${ }^{\mathrm{TM}}$ surgical system master console, allowing the surgeons to perform this operation while visualizing the images from the stereo-endoscope through the 3D display.

- Tissue Tracking: Since the tissue in the endoscopic field of view undergoes deformations and changes in position due to the highly dynamic nature of this environment, the LongTerm Safety Area Tracking (LT-SAT) algorithm [102] is used to track the SA during the surgical operation. Tracking-based and detection-based approaches are combined to improve the robustness of the SA tracking against the events mentioned above. Eventual tracking failures are detected exploiting a Bayesian inference-based approach. Redetection after failure is performed using a Model Update Strategy (MUpS) to improve the SA.

- Tissue 3D Reconstruction: In order to measure the spatial relationship between the surgical instruments and the SA, it is necessary to retrieve the 3D surface information of the tissue contained in the SA. This is done using a dense soft tissue stereo-reconstruction algorithm [103]. Since the 3D reconstruction algorithm is computationally expensive, only the area contained inside the tracked SA is considered. The algorithm is based on a block matching approach, exploiting a non-parametric Census transform to make the stereo-correspondence robust to illumination variations. Two different techniques are used to improve the density and the smoothness of the reconstructed surface. The Simple Linear Iterative Clustering (SLIC) super pixel is used to refine the disparity and fill any missing area of the image. Subsequently, another strategy is used to further smooth the result: the disparity image is considered as a 2D Laplace equation problem, where the disparity values on the contours of the super pixels are considered as the Dirichlet boundary conditions, and the remaining pixels as the initial conditions of the problem. Then, smoothing is obtained by solving the equations using the Gauss-Seidel method with red and black ordering.

- Safety Augmentation: This final step merges all information extracted in previous steps to compute the Safety Volume (SV) to be protected and to show safety warnings. The $\mathrm{SV}$ is computed as an ellipsoid fitted on the computed point cloud representing the SA. Once this volume is computed, the system allows: (i) to display its 2D projection on the images by means of $\mathrm{AR}$ in the $3 \mathrm{D}$ visor, and (ii) to warn the surgeon when the robotic 
instruments are approaching the protected tissue surface, with the aim to avoid possible injuries to the delicate area contained in it. Spatial neighbor search based on an octree structure is used to measure the distance between the robotic instrument end-effector and the reconstructed surface. A gauge located in the top-right corner of the image is displayed to warn the surgeon if the instrument-tissue distance is inside a predetermined range, as shown in Fig. 8. A multithreading approach was exploited to make the integration of the computer vision algorithms run at interactive frame rates, providing $\mathrm{AR}$ features update rate up to $4 \mathrm{fps}$, without impacting the real-time visualization of the stereo endoscopic video.

EnViSoRS was integrated into the da Vinci Research Kit (dVRK), a platform provided by Intuitive Surgical Inc. (USA) to advance research in teleoperated robotic surgical systems. Nevertheless, EnViSoRS could be integrated in any surgical robotic system equipped with a stereo-endoscope and actuated instruments. Due to its modularity, it can also be used in a simple laparoscopic setup with the requirement of having a tracking system for the instruments and endoscope.

The performance of EnViSoRS in terms of accuracy, robustness, and usability was evaluated by asking a team of surgeons and engineers to perform a simulated surgical task on a liver phantom [104], with and without the AR features provided by the system. Results show an overall accuracy in accordance with surgical requirements $(<5 \mathrm{~mm})$, and high robustness in the computation of the SV in terms of precision and recall of its identification. Qualitative results regarding the system usability indicate that it integrates well with the commercial surgical robot and has indeed potential to offer useful assistance during real surgeries.

\section{Conclusion}

In the last decades, major complications in surgery have emerged as a significant global public health issue, so as to require the practical implementation of safety measures to prevent injuries and deaths in the different phases of surgery. The introduction of novel technologies in the operating theater, such as robotic surgical operating systems, opens new questions on how much and in which way safety can be further improved. Computer-assisted surgery (CAS) has demonstrated to be able to enhance the surgeon's capabilities, by providing direct, patient and process-specific support intra-operatively to surgeons with different degrees of experience. Thus, the development of novel CAS tools exploiting the endoscope or the kinematics of the robotic arms, would represent a relevant step towards a safer clinical procedure, improving the overall quality of healthcare.

This chapter has presented the main areas involved in CAS, focusing on the application of AR during the execution of a surgical procedure. Different modalities for the selection of the anatomical target area have been presented, using either 3D models extracted from a preoperative planning or selecting the area of interest directly on the intra-operative endoscopic image. Due to the highly dynamic nature of soft tissues during surgery, the run-time surgical scene information update is fundamental to ensure reliability and robustness of the AR system. To this end, an overview of $3 \mathrm{D}$ reconstruction and tissue tracking methods was 
presented, and different modalities to represent such information and provide the surgeons with safety warnings were introduced.

Medical imaging has reached a high level of maturity in a wide variety of specific areas. However, the combination of such knowledge and its adaptation and integration to create novel applications for the clinical practice is still challenging. In fact, there are many aspects that must be considered in the development of a surgical application. The methods should ensure a high level of reliability, robustness and accuracy, since their performances can directly affect the outcome of the surgery. This aspect is often in contrast with real-time requirements, needed to transparently augment the surgical scene. Not less important is the way in which the safety applications interface with the surgeons.

As an example of recent advances in AR application in abdominal surgery, the Enhanced Vision System for Robotic Surgery (EnViSoRS) is presented. This system has demonstrated the feasibility of combining different computer vision algorithms to provide the surgeons with safety features based on AR applications. The methodological progresses made in this work stress the potential of such algorithms to extract and exploit useful information implicitly contained in surgical images, overcoming challenges related to the introduction of extra equipment in the operating room.

Overall, the main motivation of surgical safety systems is to improve the success rate of surgeries, having a positive impact on the patients' health by reducing number of complications and the risk of death. Therefore, this is an area of research with critical importance; one which will see large growth in upcoming years.

\section{References}

1. Gao Y, Tannenbaum A, Kikinis R. Simultaneous multi-object segmentation using local robust statistics and contour interaction. In International MICCAI Workshop on Medical Computer Vision; 2010. 195-203.

2. Nicolau SA, Goffin L, Soler L. A low cost and accurate guidance system for laparoscopic surgery: Validation on an abdominal phantom. In ACM symposium on Virtual Reality Software and Technology; 2005. 124-133.

3. Nicolau S, Soler L, Mutter D, Marescaux J. Augmented reality in laparoscopic surgical oncology. Surgical Oncology. 2011; 20: 189-201.

4. Pessaux P, Diana M, Soler L, Piardi T, Mutter D, Marescaux J. Towards cybernetic surgery: Robotic and augmented reality-assisted liver segmentectomy. Langenbeck's Archives of Surgery. 2015; 400: 381-385.

5. Penza V, Ortiz J, De Momi E, Forgione A, \& Mattos L. Virtual assistive system for robotic single incision laparoscopic surgery. In 4th Joint Workshop on New Technologies for Computer/Robot Assisted Surgery; 2014; Genova. 
6. Ukimura O, Gill IS. Image-fusion, augmented reality, and predictive surgical navigation. Urologic Clinics of North America. 2009; 36: 115-123.

7. Zitova B, Flusser J. Image registration methods: A survey. Image and Vision Computing. 2003; 21: 977-1000.

8. Safety WHOP, Organization WH, others. WHO Guidelines for Safe Surgery: 2009: safe surgery saves lives. 2009.

9. Weiser TG, Regenbogen SE, Thompson KD, Haynes AB, Lipsitz SR, Berry WR, et al. An estimation of the global volume of surgery: a modelling strategy based on available data.: The Lancet; 2008.

10. Haynes AB, Weiser TG, Berry WR, Lipsitz SR, Breizat AHS, Dellinger EP, et al. A surgical safety checklist to reduce morbidity and mortality in a global population: New England Journal of Medicine; 2009.

11. Tang R, Ranmuthugala G, Cunningham aF. "Surgical safety checklists: a review: ANZ journal of surgery.

12. Lam A, Kaufman Y, Khong SY, Liew A, Ford S, \& Condous G. Dealing with complications in laparoscopy. Best Practice \& Research Clinical Obstetrics \& Gynaecology. (2009; 23(5).

13. Crist DW, Gadacz aTR. Complications of laparoscopic surgery. Surgical Clinics of North America. 1993; 73(2).

14. Opitz I, Gantert W, Giger U, Kocher T, \& Krähenbühl L. Bleeding remains a major complication during laparoscopic surgery: analysis of the SALTS database. Langenbeck's archives of surgery. ; 390(2).

15. Cole AP, Trinh QD, Sood A, \& Menon M. The rise of robotic surgery in the new millennium. The Journal of urology. ; 197(2).

16. Surgical I. Intuitive Surgical Annual Report 2016. Intuitive Surgical's website; 2010.

17. Navab N, Hennersperger C, Frisch B, \& Fürst B. Personalized, relevance-based multimodal robotic imaging and augmented reality for computer assisted interventions. Medical Image Analysis. 2016; 33.

18. Bernhardt S, Nicolau SA, Soler L, Doignon C. The status of augmented reality in laparoscopic surgery as of 2016. Medical Image Analysis. 2017; 37: 66-90.

19. Pham DL, Xu C, \& Prince JL. Current methods in medical image segmentation. Annual review of biomedical engineering. 2000.

20. Heimann T, \& Meinzer HP. Statistical shape models for 3D medical image segmentation: a review. Medical image analysis. 2009; 13(4).

21. Lorensen WE, \& Cline HE. Marching cubes: A high resolution 3D surface construction algorithm. In ACM siggraph computer graphics; 1987: ACM. 136169.

22. Besl PJ, McKay ND, others. A method for registration of 3-D shapes. IEEE Transactions on Pattern Analysis and Machine Intelligence. 1992; 14: 239-256. 
23. Zhang Z. Iterative point matching for registration of free-form curves and surfaces. International Journal of Computer Vision. 1994; 13: 119-152.

24. Sotiras A, Davatzikos C, Paragios N. Deformable medical image registration: A survey. IEEE Transactions on Medical Imaging. 2013; 32: 1153-1190.

25. Sederberg TW, Parry SR. Free-form deformation of solid geometric models. ACM SIGGRAPH Computer Graphics. 1986; 20: 151-160.

26. Rueckert D, Sonoda LI, Hayes C, Hill DLG, Leach MO, Hawkes DJ. Nonrigid registration using free-form deformations: application to breast MR images. IEEE Transactions on Medical Imaging. 1999; 18: 712-721.

27. Sotiras A, Davatzikos C, Paragios N. Deformable medical image registration: A survey. IEEE Transactions on Medical Imaging. 2013; 32: 1153-1190.

28. Deshpande N, Ortiz J, Caldwell DG, \& Mattos LS. Enhanced computerassisted laser microsurgeries with a "virtual microscope" based surgical system. In IEEE International Conference on Robotics and Automation (ICRA); 2014; Hong Kong: IEEE. 4194-4199.

29. Penza V, De Momi E, Enayati N, Chupin T, Ortiz J, Mattos LS. enVisors: enhanced Vision system for robotic surgery. a User-Defined safety Volume Tracking to Minimize the risk of intraoperative Bleeding. Frontiers in Robotics and AI. 2017; 4: 15.

30. Moccia S, De Momi E, Guarnaschelli M, Savazzi M, Laborai A, Guastini L, et al. Confident texture-based laryngeal tissue classification for early stage diagnosis support. Journal of Medical Imaging. 2017; 4: 034502.

31. Moccia S, Vanone GO, De Momi E, Laborai A, Guastini L, Peretti G, et al. Learning-based classification of informative laryngoscopic frames. Computer methods and programs in biomedicine. 2018; 158: 21-30.

32. Griffiths C, Barker J, Bleiker T, Chalmers R, Creamer D. Rook's textbook of dermatology: John Wiley \& Sons; 2016.

33. Carmeliet P, Jain RK. Angiogenesis in cancer and other diseases. Nature. 2000; 407: 249-257.

34. Campochiaro PA. Molecular pathogenesis of retinal and choroidal vascular diseases. Progress in Retinal and Eye Research. 2015.

35. Moccia S, Foti S, Routray A, Prudente F, Perin A, Sekula RF, et al. Toward Improving Safety in Neurosurgery with an Active Handheld Instrument. Annals of biomedical engineering. 2018.

36. Stewart JW, Akselrod GM, Smith DR, Mikkelsen MH. Toward multispectral imaging with colloidal metasurface pixels. Advanced Materials. 2017; 29.

37. Machida H, Sano Y, Hamamoto Y, Muto M, Kozu T, Tajiri H, et al. Narrowband imaging in the diagnosis of colorectal mucosal lesions: a pilot study.

Endoscopy. 2004; 36: 1094-1098. 
38. Emsley JW, Lindon JC. NMR spectroscopy using liquid crystal solvents: Elsevier; 2018.

39. Wernick MN, Yang Y, Brankov JG, Yourganov G, Strother SC. Machine learning in medical imaging. IEEE Signal Processing Magazine. 2010; 27: 25-38.

40. Kourou K, Exarchos TP, Exarchos KP, Karamouzis MV, Fotiadis DI. Machine learning applications in cancer prognosis and prediction. Computational and Structural Biotechnology Journal. 2015; 13: 8-17.

41. Shannon CE. A mathematical theory of communication. ACM SIGMOBILE Mobile Computing and Communications Review. 2001; 5: 3-55.

42. Castellano G, Bonilha L, Li LM, Cendes F. Texture analysis of medical images. Clinical Radiology. 2004; 59: 1061-1069.

43. Guo Z, Zhang L, Zhang D. A completed modeling of local binary pattern operator for texture classification. IEEE Transactions on Image Processing. 2010; 19: 1657-1663.

44. Haralick RM. Statistical and structural approaches to texture. Proceedings of the IEEE. 1979; 67: 786-804.

45. Freeman WT, Roth M. Orientation histograms for hand gesture recognition. In International Workshop on Automatic Face and Gesture Recognition; 1995. 296301.

46. Moccia S, Mattos LS, Patrini I, Ruperti M, Poté N, Dondero F, et al. Computer-assisted liver graft steatosis assessment via learning-based texture analysis. International Journal of Computer Assisted Radiology and Surgery. 2018.

47. Misawa M, Kudo Se, Mori Y, Takeda K, Maeda Y, Kataoka S, et al. Accuracy of computer-aided diagnosis based on narrow-band imaging endocytoscopy for diagnosing colorectal lesions: comparison with experts. International Journal of Computer Assisted Radiology and Surgery. 2017;: 1-10.

48. Fu Y, Zhang W, Mandal M, Meng MQH. Computer-aided bleeding detection in WCE video. IEEE Journal of Biomedical and Health Informatics. 2014; 18: 636-642.

49. Horner JL, Gianino PD. Phase-only matched filtering. Applied Optics. 1984; 23: 812-816.

50. Walnut DF. An introduction to wavelet analysis: Springer Science \& Business Media; 2013.

51. Fraz MM, Remagnino P, Hoppe A, Uyyanonvara B, Rudnicka AR, Owen CG, et al. Blood vessel segmentation methodologies in retinal images--a survey.

Computer Methods and Programs in Biomedicine. 2012; 108: 407-433.

52. Magoulas GD. Neuronal networks and textural descriptors for automated tissue classification in endoscopy. Oncology Reports. 2006; 15: 997-1000. 
53. Burt P, Adelson E. The Laplacian pyramid as a compact image code. IEEE Transactions on Communications. 1983; 31: 532-540.

54. Kumar S, Saxena R, Singh K. Fractional Fourier transform and fractional-order calculus-based image edge detection. Circuits, Systems, and Signal Processing. 2017; 36: 1493-1513.

55. Esteva A, Kuprel B, Novoa RA, Ko J, Swetter SM, Blau HM, et al. Dermatologist-level classification of skin cancer with deep neural networks. Nature. 2017; 542: 115-118.

56. Poplin R, Varadarajan AV, Blumer K, Liu Y, McConnell MV, Corrado GS, et al. Prediction of cardiovascular risk factors from retinal fundus photographs via deep learning. Nature Biomedical Engineering. 2018;: 1.

57. Nanni L, Ghidoni S, Brahnam S. Handcrafted vs Non-Handcrafted Features for computer vision classification. Pattern Recognition. 2017.

58. Kotsiantis SB, Zaharakis I, Pintelas P. Supervised machine learning: A review of classification techniques. 2007..

59. McCallum A, Nigam K, others. A comparison of event models for naive Bayes text classification. In ICML/AAAI Workshop on Learning for Text Categorization; 1998. 41-48.

60. Friedman N, Geiger D, Goldszmidt M. Bayesian network classifiers. Machine Learning. 1997; 29: 131-163.

61. Ramoni M, Sebastiani P. Robust Bayes classifiers. Artificial Intelligence. 2001; 125: 209-226.

62. Dasarathy BV. Nearest neighbor () norms: pattern classification techniques. 1991.

63. Vrooman HA, Cocosco CA, Lijn F, Stokking R, Ikram MA, Vernooij MW, et al. Multi-spectral brain tissue segmentation using automatically trained k-NearestNeighbor classification. Neuroimage. 2007; 37: 71-81.

64. Berens J, Mackiewicz M, Bell D. Stomach, intestine, and colon tissue discriminators for wireless capsule endoscopy images. In SPIE Medical Imaging; 2005. 283-291.

65. Rosenblatt F. The perceptron: A probabilistic model for information storage and organization in the brain. Psychological Review. 1958; 65: 386.

66. Magoulas GD. Neuronal networks and textural descriptors for automated tissue classification in endoscopy. Oncology Reports. 2006; 15: 997-1000.

67. Karargyris A, Bourbakis N. Wireless capsule endoscopy and endoscopic imaging: A survey on various methodologies presented. IEEE Engineering in Medicine and Biology Magazine. 2010; 29: 72-83.

68. Shen L, Rangayyan RM, Desautels JEL. Detection and classification of mammographic calcifications. International Journal of Pattern Recognition and Artificial Intelligence. 1993; 7: 1403-1416. 
69. Safavian SR, Landgrebe D. A survey of decision tree classifier methodology. IEEE transactions on systems, man, and cybernetics. 1991; 21: 660-674.

70. Weiss SM, Indurkhya N. Rule-based machine learning methods for functional prediction. Journal of Artificial Intelligence Research. 1995; 3: 383-403.

71. Cortes C, Vapnik V. Support vector machine. Machine Learning. 1995; 20 : 273-297.

72. Moccia S, Wirkert SJ, Kenngott H, Vemuri AS, Apitz M, Mayer B, et al. Uncertainty-Aware Organ Classification for Surgical Data Science Applications in Laparoscopy. IEEE Transactions on Biomedical Engineering. 2018.

73. Mukherjee R, Manohar DD, Das DK, Achar A, Mitra A, Chakraborty C. Automated tissue classification framework for reproducible chronic wound assessment. BioMed Research International. 2014; 2014.

74. Bernal J, Tajkbaksh N, Sánchez FJ, Matuszewski BJ, Chen H, Yu L, et al. Comparative validation of polyp detection methods in video colonoscopy: Results from the MICCAI 2015 endoscopic vision challenge. IEEE Transactions on Medical Imaging. 2017; 36: 1231-1249.

75. Moccia S, De Momi E, El Hadji S, Mattos LS. Blood vessel segmentation algorithms--Review of methods, datasets and evaluation metrics. Computer methods and programs in biomedicine. 2018; 158: 71-91.

76. Penza V, Moccia S, Gallarello A, Panaccio A, De Momi E, Mattos LS. Context-Aware Augmented Reality for Laparoscopy. In Sixth national Congress of Bioengineering; 2018; Milan.

77. Bergen T, Wittenberg T. Stitching and Surface Reconstruction from Endoscopic Image Sequences: A Review of Applications and Methods. 2014.

78. Röhl S, Bodenstedt S, Suwelack S, Kenngott H, Müller-Stich BP, Dillmann R, et al. Dense GPU-enhanced surface reconstruction from stereo endoscopic images for intraoperative registration. Medical physics. 2012; 39: 1632-1645.

79. Mountney P, Stoyanov D, \& Yang GZ. Three-dimensional tissue deformation recovery and tracking. IEEE Signal Processing Magazine. 2010 July: 14-24.

80. Mirota DJ, Ishii M, Hager GD. Vision-based navigation in image-guided interventions. Annual review of biomedical engineering. 2011; 13: 297-319.

81. Bernhardt S, Abi-Nahed J, \& Abugharbieh R. Robust dense endoscopic stereo reconstruction for minimally invasive surgery. In International MICCAI Workshop on Medical Computer Vision; 2012; Heidelberg. 254-262.

82. Stoyanov D, Scarzanella MV, Pratt P,\&YGZ. Real-time stereo reconstruction in robotically assisted minimally invasive surgery. In International Conference on Medical Image Computing and Computer-Assisted Intervention; 2010; Heidelberg. 275-282. 
83. Röhl S, Bodenstedt S, Suwelack S, Kenngott H, Müller-Stich BP, Dillmann R, et al. Dense GPU-enhanced surface reconstruction from stereo endoscopic images for intraoperative registration. Medical physics. 2012; 39(3).

84. Mountney P, Stoyanov D, Davison A, \& Yang GZ. Simultaneous stereoscope localization and soft-tissue mapping for minimal invasive surgery. In International Conference on Medical Image Computing and Computer-Assisted Intervention; 2006; Heidelberg. 347-354.

85. Mountney P, Yang aGZ. Motion compensated SLAM for image guided surgery. In International Conference on Medical Image Computing and ComputerAssisted Intervention.; 2010; Heidelberg. 496-504.

86. Maier-Hein L, Mountney P, Bartoli A, Elhawary H, Elson D, Groch A, et al. Optical techniques for 3D surface reconstruction in computer-assisted laparoscopic surgery. Medical image analysis. 2013; 17: 974-996.

87. Maier-Hein L, Groch A, Bartoli A, Bodenstedt S, Boissonnat G, Chang PL, et al. Comparative validation of single-shot optical techniques for laparoscopic 3-D surface reconstruction. IEEE transactions on medical imaging. 2014; 33(10).

88. Scharstein D, Szeliski R. A taxonomy and evaluation of dense two-frame stereo correspondence algorithms. International journal of computer vision. 2002; 47: 742.

89. Brown MZ, Burschka D, \& Hager GD. Advances in computational stereo. IEEE transactions on pattern analysis and machine intelligence. 2003; 25(8): 993 1008.

90. Tian Q, Huhns MN. Algorithms for subpixel registration. Computer Vision, Graphics, and Image Processing. 1986; 35: 220-233.

91. Stoyanov D. Surgical vision. Annals of biomedical engineering. 2012; 40: 332345.

92. Yang H, Shao L, Zheng F, Wang L, Song Z. Recent advances and trends in visual tracking: A review. Neurocomputing. 2011; 74: 3823-3831.

93. Lowe DG. Distinctive image features from scale-invariant keypoints. International journal of computer vision. 2004; 60: 91-110.

94. Viola P, Jones M. Rapid object detection using a boosted cascade of simple features. In Computer Vision and Pattern Recognition, 2001. CVPR 2001. Proceedings of the 2001 IEEE Computer Society Conference on; 2001. I--I.

95. Fichera L. Realization of a Cognitive Supervisory System for Laser Microsurgery. Cognitive Supervision for Robot-Assisted Minimally Invasive Laser Surgery. 2016;: 79-88.

96. Rosenberg LB. Virtual fixtures: Perceptual tools for telerobotic manipulation. In IEEE Virtual Reality Annual International Symposium; 1993. 76-82.

97. Bowyer SA, Davies BL, Baena FR. Active constraints/virtual fixtures: A survey. IEEE Transactions on Robotics. 2014; 30: 138-157. 
98. Enayati N, Costa ECA, Ferrigno G, De Momi E. A dynamic non-energystoring guidance constraint with motion redirection for robot-assisted surgery. In EEE/RSJ International Conference on Intelligent Robots and Systems; 2016. 4311-4316.

99. Olivieri E, Barresi G, Caldwell DG, Mattos LS. Haptic Feedback for Control and Active Constraints in Contactless Laser Surgery: Concept, Implementation, and Evaluation. IEEE Transactions on Haptics. 2018; 11: 241-254.

100. Vitrani MA, Poquet C, Morel G. Applying virtual fixtures to the distal end of a minimally invasive surgery instrument. IEEE Transactions on Robotics. 2017; 33: 114-123.

101. Tauscher S, Fuchs A, Baier F, Kahrs LA, Ortmaier T. High-accuracy drilling with an image guided light weight robot: Autonomous versus intuitive feed control. International Journal of Computer Assisted Radiology and Surgery. 2017; 12: $1763-1773$.

102. Penza V, Du X, Stoyanov D, Forgione A, Mattos LS, \& De Momi E. Long Term Safety Area Tracking (LT-SAT) with online failure detection and recovery for robotic minimally invasive surgery. Medical image analysis. 2018; 45.

103. Penza V, Ortiz J, Mattos LS, Forgione A, De Momi E. Dense soft tissue 3D reconstruction refined with super-pixel segmentation for robotic abdominal surgery. International Journal of Computer Assisted Radiology and Surgery. 2016; 11: 197-206.

104. Penza V, Ciullo AS, Moccia S, Mattos LS, \& De Momi E. EndoAbS dataset: Endoscopic abdominal stereo image dataset for benchmarking 3D stereo reconstruction algorithms. The International Journal of Medical Robotics and Computer Assisted Surgery. 2018; e1926. 